\title{
THE ENDOMORPHISM NEAR-RINGS OF THE SYMMETRIC GROUPS OF DEGREE AT LEAST FIVE
}

\author{
Y. FONG and J. D. P. MELDRUM \\ (Received 17 September 1979; revised 11 February 1980) \\ Communicated by D. E. Taylor
}

\begin{abstract}
The near-ring distributively generated by the semigroup of all endomorphisms of $S_{n}$, the symmetric group of degree $n$, for $n \geqslant 5$, is close to being the near-ring of all mappings from $S_{n}$ to itself respecting the identity. In this paper, the structure of these near-rings is studied in detail. In particular, addition and multiplication rules for the elements given in canonical form are determined. A complete list of all right ideals, left ideals, right invariant and left invariant subgroups is given.
\end{abstract}

1980 Mathematics subject classification (Amer. Math. Soc.): 16 A 76.

\section{Introduction}

A near-ring is a set $R$ with two operations + and $; \operatorname{such}$ that $(R,+)$ is a group, $(R, \cdot)$ is a semigroup and the left distributive law is satisfied : $x(y+z)=x y+x z$ for all $x, y, z \in R$. In general the extra axiom $0 x=0$ for all $x \in R$ is imposed to give a zerosymmetric near-ring. An element $s \in R$ is called distributive if $(x+y) s=x s+y s$ for all $x, y \in R$. The set of distributive elements forms a multiplicative semigroup. If there exists a multiplicative semigroup of distributive elements $S$ such that $R=\mathrm{Gp}\langle S\rangle$, we say that $R$ is a distributively generated (d.g.) near-ring, often denoted $(R, S)$. The typical example of a near-ring is $M(G)$, the set of all mappings from a group $G$ to itself with pointwise addition and composition of mappings. $M_{0}(G)=\left\{\alpha \in M(G) ; 0_{G} \alpha=0_{G}\right\}$ is the typical example of a zero-symmetric near-ring. The distributive elements of $M(G)$ are End $G$, the semigroup of endomorphisms of $G$. $(E(G)$, End $G)$, the d.g. near-ring generated by End $G$ is of special interest, as are $(I(G), \operatorname{Inn} G),(A(G)$, Aut $G)$, the d.g. near-rings generated by $\operatorname{Inn} G$, the inner automorphisms of $G$, and Aut $G$, all the automorphisms of $G$. 
The structure of $E(G), A(G)$ and $I(G)$ has been studied for many groups or classes of groups (see the list of references at the end of this paper). This paper presents an extension of some of the results of Fong (1979), namely the detailed structure of $E(G)$ for $G=S_{n}, n \geqslant 5$, the symmetric group of degree at least 5. This extends considerably the work in Meldrum (1978). The interest in such detailed work is in providing d.g. near-rings whose structure is known in great detail, in comparing the structure with that of $M_{0}(G)$ for an arbitrary group $G$, and in providing the necessary details of the addition and multiplication tables for anyone wishing to study such d.g. near-rings further. For general results and ideas we refer to Pilz (1977). The main difference is that we use left rather than right near-rings. The notation has been changed from Fong (1979) to make it more self-consistent.

The first author would like to acknowledge financial support from the University of Edinburgh in the form of a Postgraduate Studentship awarded for the year 1978-79.

\section{Addition and multiplication tables}

For the rest of the paper, we write $G$ for $S_{n}$, the symmetric group of degree $n, n \geqslant 5$, $H$ for $A_{n}$, the alternating group of degree $n, S$ for End $G, R$ for $E(G)$. We now quote the following result from Meldrum (1978), Theorem 4.11, using our notation.

THEOREM 2.1. Let $n \geqslant 5$. Then

$$
I(G)=A(G)=E(G)=R
$$

and $R$ has an ideal $N$ such that

$$
\begin{aligned}
N^{2} & =\{0\}, \\
R / N & \cong M_{0}(H) \oplus Z_{2}, \\
N & =\left\{\alpha \in M_{0}(G) ; G \alpha \subseteq H\right\} .
\end{aligned}
$$

$\oplus$ indicates direct sum, $Z_{\mathbf{2}}$ the ring of integers modulo 2 . We will enumerate the elements of $G$ as follows :

$$
H=\left\{0=g_{0}, \ldots, g_{m}\right\}, \quad G-H=\left\{g_{m+1}, \ldots, g_{2 m+1}\right\},
$$

where

$$
1+m=n ! / 2, \quad g_{m+i+1}=g_{i}+(12), \quad \text { for } 0 \leqslant i \leqslant m .
$$

Using the results of Heatherly (1972) or Pilz (1977), we can write $M_{0}(H)$ as a direct sum of right ideals as follows :

$$
M_{0}(H)=\sum_{i=1}^{m} \varepsilon_{i} M_{0}(H)
$$


where $\varepsilon_{i}$ maps $g_{i}$ to itself and $H-\left\{g_{i}\right\}$ to zero. Then $\left(\varepsilon_{i} M_{0}(H),+\right) \cong(H,+)$ and $\varepsilon_{i} M_{0}(H)=\operatorname{Ann}\left(H-\left\{g_{i}\right\}\right)$ is the annihilator of $H-\left\{g_{i}\right\}$. Using a similar method it is obvious that we have a decomposition for $N$ as a sum of right ideals,

$$
N=\sum_{i=1}^{m+1} \delta_{i} M_{0}(H)
$$

where $\delta_{i}$ maps $g_{i+m}$ to $g_{1}$ and $G-\left\{g_{i+m}\right\}$ to zero. Then

$$
\left(\delta_{i} M_{0}(H),+\right) \cong(H,+) \quad \text { and } \delta_{i} M_{0}(H)=N \cap \operatorname{Ann}\left(G-\left\{g_{i+m}\right\}\right) .
$$

We will use another notation for $\delta_{i} M_{0}(H)$ :

$$
\delta_{i} M_{0}(H)=\left\{\eta_{i j} ; 0 \leqslant j \leqslant m\right\},
$$

where $\eta_{i j}$ maps $g_{m+i}$ to $g_{j}$. We have been tacitly assuming that $M_{0}(H) \subseteq R$. We now show that this is the case up to isomorphism.

LEMMA 2.2. $R$ contains a subnear-ring which is isomorphic to $M_{0}(H)$, and acts in the natural way on $H$, annihilating $G-H$.

Proof. Let $\alpha \in M_{0}(H)$. By Theorem 2.1, we can choose $\bar{\alpha} \in R$ such that $\bar{\alpha}$ has the same effect as $\alpha$ on $H$, and $G \bar{\alpha} \subseteq H$. Let $g_{m+i} \bar{\alpha}=g_{j(i)}, 1 \leqslant i \leqslant m+1$. Then $\eta=\sum_{i=1}^{m+1} \eta_{i j(i)}$ satisfies the following:

$$
\begin{aligned}
(G-H)(\bar{\alpha}-\eta) & =0, \\
g_{i}(\bar{\alpha}-\eta) & =g_{i} \alpha, \quad 0 \leqslant i \leqslant m .
\end{aligned}
$$

This suffices to prove the result.

So we can without loss of generality assume that $M_{0}(H) \subseteq R$, by identifying it with its isomorphic copy. We can now write $R$ as a sum of three subgroups.

$$
R=N+M_{0}(H)+Z_{2}
$$

where $N$ is an ideal, $M_{0}(H)$ and $Z_{2}$ are subnear-rings, $R / N \cong M_{0}(H) \oplus Z_{2}$, and $Z_{2}=\{0, \theta\}$, where $\theta \in$ End $G$ maps $G-H$ to $(12)$ and $H$ to zero. $M_{0}(H)$ and $Z_{2}$ together generate $M_{0}(H) \oplus Z_{2}$ and $R$ is a split extension of $N$ by $M_{0}(H) \oplus Z_{2}$, that is, $R=\left(M_{0}(H) \oplus Z_{2}\right)+N, N \cap\left(M_{0}(H) \oplus Z_{2}\right)=0$ and $N$ is a normal subgroup of $R$. From (2.4) we can represent an arbitrary element of $R$ as $\eta+\beta+\alpha$ where $\eta \in N$, $\beta \in M_{0}(H)$ and $\alpha \in Z_{2}$, or $(\eta, \beta, \alpha)$. From (2.2), we can write $\eta$ in the form $\sum_{i=1}^{m+1} \delta_{i} \xi_{i}$, where $\xi_{i} \in M_{0}(H)$. From (2.3), we can write $\eta=\sum_{i=1}^{m+1} \eta_{i j(i)}$. We will use either form as appropriate.

LEMMA 2.3. For each $i, 1 \leqslant i \leqslant m+1$,

$$
\mathrm{Gp}\left\langle\delta_{i} M_{0}(H), \theta\right\rangle \cong G,
$$


where the isomorphism is given by $\eta_{i j} \rightarrow g_{j}, \theta \rightarrow(12)$.

This result can be proved by routine checks. We can now give the addition and multiplication rules for $R$.

\section{THEOREM 2.4 .}

(a) $(\eta, \beta, \alpha)+\left(\eta^{\prime}, \beta^{\prime}, \alpha^{\prime}\right)=\left(\eta+\eta^{\prime \alpha}, \beta+\beta^{\prime}, \alpha+\alpha^{\prime}\right)$

(b) $\left(\sum_{i=1}^{m+1} \delta_{i} \xi_{i}, \beta, 0\right)\left(\eta^{\prime}, \beta^{\prime}, \alpha^{\prime}\right)=\left(\sum_{i=1}^{m+1} \delta_{i} \xi_{i} \beta^{\prime}, \beta \beta^{\prime}, 0\right)$

(c) $\left(\sum_{i=1}^{m+1} \eta_{i j(i)}, \beta, \theta\right)\left(\sum_{i=1}^{m+1} \delta_{i} \xi_{i}, \beta^{\prime}, \alpha\right)=\left(\sum_{i=1}^{m+1} \delta_{i} \xi_{j(i)+1}, \beta \beta^{\prime}, \alpha\right)$.

ProOF. (a) $\eta^{\prime \alpha}=-\alpha+\eta^{\prime}+\alpha$ is an element of $N$ as can easily be checked. The proof is easy, particularly if it is separated into two cases : the action on $H$ and the action on $G-H$.

(b) is straightforward, again using the two cases as in (a) and remembering that $g_{m+i} \delta_{i} \xi_{i} \in H$.

(c) is straightforward, except possibly for the action on $G-H$. So let $g_{m+k} \in G-H$. Then

$$
\begin{aligned}
g_{m+k}\left(\sum_{i=1}^{m+1} \eta_{i j(i)}, \beta, \theta\right) & =g_{j(k)}+(12)=g_{j(k)+m+1}, \\
g_{m+j(k)+1}\left(\sum_{i=1}^{m+1} \delta_{i} \xi_{i}, \beta^{\prime}, \alpha\right) & =g_{1} \xi_{j(k)+1}+g_{m+j(k)+1} \alpha \\
& =g_{m+k}\left(\sum_{i=1}^{m+1} \delta_{i} \xi_{j(i)+1}, \beta \beta^{\prime}, \alpha\right) .
\end{aligned}
$$

This is enough to prove the result.

Note that in $\eta_{i j}$ we have $0 \leqslant j(i) \leqslant m$. So $1 \leqslant j(i)+1 \leqslant m+1$, which shows that no problem of definition can occur in (c).

\section{The right ideals of $R$}

We first obtain some results about normal subgroups which narrow down possibilities quite considerably. From a standard result in group theory (Scott (1964)) the normal subgroups of $S_{n}$ for $n \geqslant 5$ are $0, A_{n}, S_{n}$. The following result is an easy corollary of this.

LEMMA 3.1. Let $K$ be a normal subgroup of $(R,+)$. If $\theta \in K$, then $N \subseteq K$. 
ProOF. By Lemma $2.3,0 \in K$ implies $\delta_{i} M_{0}(H) \subseteq K, 1 \leqslant i \leqslant m+1$, since the only normal subgroups of $S_{n}, n \geqslant 5$, are $0, A_{n}$ and $S_{n}$.

Lemma 3.2. Let $K$ be a normal subgroup of $N+M_{0}(H)$. Then

$$
K=\sum_{j \in J} \delta_{j} M_{0}(H)+\sum_{i \in I} \varepsilon_{i} M_{0}(H)
$$

for some subsets $J$ of $\{1,2, \ldots, m+1\}$ and $I$ of $\{1, \ldots, m\}$.

Proof. This result follows easily from the following facts. $\delta_{j} M_{0}(H)$ and $\varepsilon_{i} M_{0}(H)$ are both isomorphic to $H$ as groups and $H$ is a simple nonabelian group. So $N+M_{0}(H)$ is a direct sum of simple nonabelian groups, and any normal subgroup of it is simply a direct sum of a suitable collection of the factors (Scott (1964)). This proves the result.

THEOREM 3.3. The following is a complete list of right ideals of $R$ :

$$
\sum_{j \in J} \delta_{j} M_{0}(H)+\sum_{i \in I} \varepsilon_{i} M_{0}(H), \quad \sum_{i \in I} \varepsilon_{i} M_{0}(H)+N+Z_{2},
$$

where $J \subseteq\{1,2, \ldots, m+1\}, I \subseteq\{1, \ldots, m\}$.

ProOf. By Lemmas 3.1 and 3.2, the above is a complete list of normal subgroups of $(R,+)$. A right ideal of a d.g. near-ring is a normal subgroup closed under right multiplication. So the rest follows from Theorem 2.4 and a description of the right ideals of $M_{0}(H)$ obtainable from Heatherly (1972) or Pilz (1977).

COROLLARY 3.4. All right ideals of $R$ are annihilators of suitable subsets of $G$ except for $N+K, K \subseteq M_{0}(H)$.

Proof. If $J \subset\{1, \ldots, m+1\}$ then

$$
\sum_{j \in J} \delta_{j} M_{0}(H)+\sum_{i \in I} \varepsilon_{i} M_{0}(H)=\operatorname{Ann}\left(G-\left(\bigcup_{j \in J} g_{m+j}\right)-\left(\bigcup_{i \in I} g_{i}\right)\right) .
$$

But if $X \subseteq H$, then $\theta \in \operatorname{Ann}(X)$. This proves the result, as Ann $\left\{g_{i} ; i \in I^{\prime}\right\}$, $I^{\prime} \subseteq\{1, \ldots, m\}$ is $N+Z_{2}+\sum_{i \in I} \varepsilon_{i} M_{0}(H)$ where $I \cup I^{\prime}=\{1, \ldots, m\}, I \cap I^{\prime}=\emptyset$.

Note that this corrects a result falsely stated in Fong (1979).

\section{The left ideals of $R$}

We start by quoting the result due to Heatherly (1972). 
LEMMA 4.1. The only left ideals of $M_{0}(H)$ are $\{0\}$ and $M_{0}(H)$.

This enables us to obtain a complete list of left ideals of $R$.

THEOREM 4.2. The following is a complete list of left ideals of $R$ :

$$
\{0\}, \quad N, \quad N+Z_{2}, \quad M_{0}(H)+N \text { and } R \text {. }
$$

ProOF. From the description of $R$ given in Section 2, it is known that these are all ideals of $R$, hence left ideals.

Suppose $K$ is a left ideal of $R$. Then $K \cap M_{0}(H)>\{0\}$ forces $M_{0}(H) \subseteq K$ by Lemma 4.1. By Lemma 3.1, if $\theta \in K$, then $N \subseteq K$, as $K$ is a normal subgroup of $R$. From the list given in Theorem 3.3, which is also a list of all normal subgroups of $R$, the only possibilities left are

$$
\sum_{j \in J} \delta_{j} M_{0}(H), \quad \sum_{j \in J} \delta_{j} M_{0}(H)+M_{0}(H)
$$

where $J \subset\{1,2, \ldots, m+1\}$. From Theorem 2.4(c), it is obvious that if $J \neq \emptyset$ then $N \subseteq K$. So we have the possibilities $N, N+M_{0}(H)$ and $M_{0}(H)$. To finish the proof we need to show that $M_{0}(H)$ is not a left ideal. But this follows immediately from Theorem 2.4(b).

Corollary 4.3. All left ideals of $R$ are two-sided ideals. Hence the list in Theorem 4.2 is a complete list of all ideals of $R$.

\section{The right $R$-subgroups of $R$}

There is not an easy description of all right $R$-subgroups of $R$, that is subgroups $K$ of $R$ such that $K R \subseteq K$. But there is a reasonably nice classification of monogenic (one-generatcr) right $R$-subgroups. If $r \in R$ then $r R$ is the right $R$-subgroup generated by $r$. We look at the different forms the element $r$ can take.

Consider an element of the form $\eta+\beta$. Using (2.1) and (2.2) we write it in the form

$$
\eta+\beta=\sum_{i \in I} \delta_{i} \xi_{i}+\sum_{j \in J} \varepsilon_{j} \zeta_{j}
$$

Here one of $I, J$ may be empty, but we assume that $I \cup J>\phi$ to avoid trivialities. This means that $\eta+\beta$ acts nontrivially precisely on $\left\{g_{j}, g_{i+m} ; j \in J, i \in I\right\}=X$ say. We now divide $X$ into $q$ equivalence classes as follows : $g_{k} \in C_{l}$ if and only if $g_{k}(\eta+\beta)=g^{\prime}$ and $g^{\prime}$ is determined uniquely by $C_{l}$. So $C_{l}$ consists of all elements in $X$ which get mapped by $\eta+\beta$ into a given element of $G$. It is easy to check that this determines a partition of $X$ which we denote $C_{1}, \ldots, C_{q}$. Denote the corresponding 
images by $h_{1}, \ldots, h_{q}$ where $h_{k}$ corresponds to $C_{k}$. By post-multiplication by elements of $M_{0}(H)$, it is obvious that we can map $h_{1}, \ldots, h_{q}$ to any $q$ elements of $H$. Note $h_{1}, \ldots, h_{q}$ all lie in $H$ by definition of $N$ and $M_{0}(H)$. Let $\varepsilon_{k}^{\prime}$ denote the element of $M_{0}(H)$ which maps $h_{k} \rightarrow h_{k}$ and $H-\left\{h_{k}\right\}$ to 0 . Then $(\eta+\beta) \varepsilon_{k}^{\prime}$ maps $C_{k}$ to $h_{k}$ and the rest to zero. It is easy to check that $\left((\eta+\beta) \varepsilon_{k}^{\prime} M_{0}(H),+\right)$ is isomorphic to $H$ in the natural way, and $(\eta+\beta) M_{0}(H)$ is isomorphic as a group to $q$ copies of $H$, namely

$$
(\eta+\beta) M_{0}(H)=\sum_{k=1}^{q}(\eta+\beta) \varepsilon_{k}^{\prime} M_{0}(H) .
$$

We put this together in the following result.

THEOREM 5.1. Let $\eta+\beta \in R$. Then the right $R$-subgroup generated by $\eta+\beta$ is isomorphic to a direct sum of $q$ copies of $H$ as given in (5.2), and $q$ is defined above.

COROLlary 5.2. The right $R$-subgroup $(\eta+\beta) M_{0}(H)$ is a right ideal if and only if each $C_{k}$ is a singleton.

Proof. Most of Theorem 5.1 has been proved already. The result follows from the observation that $(\eta+\beta) N=(\eta+\beta) \theta=0$, and so $(\eta+\beta) R=(\eta+\beta) M_{0}(H)$. Corollary 5.2 follows from the argument above and Lemma 3.2.

The next case is the $R$-subgroup $\theta R$.

THEOREM 5.3. The right $R$-subgroup $\theta R$ is isomorphic to $G$ as a group and can be given as $\left\{\theta_{x} ; x \in G\right\}$ where $(G-H) \theta_{x}=x, H \theta_{x}=0$ and $\theta_{x}+\theta_{y}=\theta_{x+y}, \theta_{x} \theta_{y}=\theta_{y}$ if $x \notin H, \theta_{x} \theta_{y}=0$ if $x \in H$.

ProOf. $(G-H) \theta=(12), H \theta=0$ by definition of $\theta$. Given $x \in G$, there exists $\varphi_{x} \in R$ such that (12) $\varphi_{x}=x$, as can easily be seen from Theorem 2.1. Then put $\theta_{x}=\theta \varphi_{x}$ and the rest follows easily.

The final case is the $R$-subgroup $(\eta+\beta+\theta) R$ where $\eta+\beta \neq 0$. We use the formula (5.1) for $\eta+\beta$ and write $X=\left\{g_{j}, g_{i+m} ; j \in J, i \in I\right\}$ again. But we need a different equivalence relation to that used for Theorem 5.1. We partition $X \cap H=\left\{g_{j} ; j \in J\right\}$ into $D_{1} \cup \ldots \cup D_{k}$ where $D_{k}$ consists of all elements of $X \cap H$ which get mapped by $\eta+\beta$ to a given element $h_{k}$ of $H$. Similarly we partition $G-H$ into $D_{u+1} \cup \ldots \cup D_{u+v}$ where $D_{u+l}$ consists of all elements of $G-H$ which are mapped by $\eta+\beta+\theta$ to a given element $g_{1}^{\prime}$ of $G-H$. Note that $v \geqslant 1$ whether or not $I$ is empty. From the fact that $\beta$ and $\eta+\theta$ act on mutually disjoint subsets, it follows that $(\eta+\beta+\theta) R=\beta R+(\eta+\theta) R$. The structure of $\beta R$ is given as a special case of Theorem 5.1. So consider

$$
(\eta+\theta) R=(\eta+\theta)\left(M_{0}(H)+N+Z_{2}\right)=(\eta+\theta) N+(\eta+\theta) Z_{2}
$$


since $G(\eta+\theta) \subseteq G-H$ and $(G-H) M_{0}(H)=0$. Define $\delta_{l}^{\prime}$ by $g_{l}^{\prime} \delta_{l}^{\prime}=g_{l}^{\prime}$ and $\left(G-\left\{g_{l}^{\prime}\right\}\right) \delta_{l}^{\prime}=0$. Then $\left((\eta+\theta) \delta_{l}^{\prime} N,+\right) \cong H$ and

$$
(\eta+\theta) N=(\eta+\theta)\left(\delta_{1}^{\prime}+\ldots+\delta_{v}^{\prime}\right) N=\sum_{l=1}^{v}(\eta+\theta) \delta_{l}^{\prime} N
$$

is the sum of $v$ copies of $H .(\eta+\theta) Z_{2}=(\eta+\theta) \theta=\theta$ and $\operatorname{so}(\eta+\theta) Z_{2}$ is a cyclic group of order 2. Finally if we consider $(\eta+\theta) \delta_{l}^{\prime} N+(\eta+\theta) Z_{2}$, it is isomorphic to $G$ as an additive group, the proof of this being similar to that of Lemma 2.3. This gives us our final case.

ThEOREM 5.4. The right $R$-subgroup $(\eta+\beta+\theta) R$ is isomorphic to the direct sum of $u$ copies of $H$ together with the sum of $v$ copies of $H$ extended by a cyclic group of order 2 :

$$
\left.(\eta+\beta+\theta) R=\sum_{k=1}^{u} \varepsilon_{k}^{\prime} M_{0}(H) \oplus\left(\left(\sum_{l=1}^{v} \delta_{l}^{\prime} N\right)+Z_{2}\right)\right)
$$

and $\delta_{l}^{\prime} N+Z_{2}$ is isomorphic to $G$ for each $l, 1 \leqslant l \leqslant v$. If $\beta=0$ then $u=0$, but $v \geqslant 1$ in all cases.

COROLlaRY 5.5. The right $R$-subgroup described in Theorem 5.4 is a right ideal only if $v=m+1$ and each $D_{k}, 1 \leqslant k \leqslant u$, is a singleton.

ProOF. This follows easily from Lemmas 3.1 and 3.2 .

The structure of general right $R$-subgroups is not so easy. Perhaps the best description is to say that it is the set theoretic union of all the monogenic right $R$ subgroups which it contains. We give here another description.

THEOREM 5.6. Let $K$ be a right $R$-subgroup of $R$. If $K \subseteq N+M_{0}(H)$, then it is the direct sum of a number of copies of $H$. If $K \nsubseteq N+M_{0}(H)$, then

$$
K \cong \sum_{i \in I} H_{i} \oplus\left(\sum_{j \in J} H_{j}+Z_{2}\right)
$$

where $H_{i} \cong H, H_{j} \cong H, H_{j}+Z_{2}$ is isomorphic to $G, I$ has at most melements and $J$ has at most $m+1$ elements.

This follows immediately from the remarks above. In fact, by induction on the number of generators, we can describe $K$ as follows. If $K \subseteq N+M_{0}(H)$, then $G$ can be expressed as a disjoint union of subsets $G=\bigcup_{i=1}^{u+v} D_{i}, u \geqslant 1, v \geqslant 0$, every element in a set $D_{i}$ is mapped to the same element, $g_{i}^{\prime} \in H$ say, which can be chosen arbitrarily for $1 \leqslant i \leqslant u$, and for $i>u, g_{i}^{\prime}$ is a word in $\left\{g_{1}^{\prime}, \ldots, g_{u}^{\prime}\right\}$. If $K \nsubseteq N+M_{0}(H)$, then $G$ can be expressed as a disjoint union of subsets

$$
G=\bigcup_{i=1}^{u+v+w} D_{i}, \quad v \geqslant 1, \quad u, \quad w \geqslant 0, \quad \bigcup_{i=1}^{u} D_{i} \subseteq H, \bigcup_{i=u+1}^{u+v} D_{i} \subseteq G-H,
$$


every element in a set $D_{i}$ is mapped to the same element, $g_{i}^{\prime}$ say, $g_{i}^{\prime} \in H$ for $1 \leqslant i \leqslant u$, $g_{i}^{\prime} \in G$ for $u+1 \leqslant i \leqslant u+v$, and each $g_{i}^{\prime}$ can be chosen arbitrarily for $1 \leqslant i \leqslant u+v$, and for $i>u+v, g_{i}^{\prime}$ is a word in $\left\{g_{1}^{\prime}, \ldots, g_{u}^{\prime}\right\}$ or in $\left\{g_{u+1}^{\prime}, \ldots, g_{u+v}^{\prime}\right\}$. In terms of Theorem $5.6,|I|=u,|J|=v$ in the second case.

\section{The left $R$-subgroups of $R$}

This case proves to be easier than the case of right $R$-subgroups. We quote the following result about left $R$-subgroups of $M_{0}(H)$.

THEOREM 6.1. The left $R$-subgroups of $M_{0}(H)$ are in 1-1 correspondence with the subgroups of $H$ and each left $R$-subgroup consists of all maps from $H$ into the corresponding subgroup of $H$.

This can be found in Laxton (1963), Betsch (1973) or Pilz (1977). The results for $R$ are remarkably similar. We first give a set of multiplications based on Theorem 2.4 , which clarify the position as regards left $R$-subgroups.

\section{LEMMA 6.2 .}

(i) $(\eta, \beta, 0) \theta=0,(\eta, \beta, \theta) \theta=\theta$.

(ii) $\left(\sum_{i=1}^{m+1} \delta_{i} \xi_{i}, \beta^{\prime}, 0\right) \beta=\left(\sum_{i=1}^{m+1} \delta_{i} \xi_{i} \beta, \beta^{\prime} \beta, 0\right)$

$\left(\eta, \beta^{\prime}, \theta\right) \beta=\left(0, \beta^{\prime} \beta, 0\right)$.

(iii) $\left(\eta^{\prime}, \beta^{\prime}, 0\right) \eta=0$,

$$
\left(\sum_{i=1}^{m+1} \eta_{i j(i)}, \beta^{\prime}, \theta\right)\left(\sum_{i=1}^{m+1} \delta_{i} \xi_{i}\right)=\left(\sum_{i=1}^{m+1} \delta_{i} \xi_{j(i)+1}, 0,0\right) .
$$

(iv) $\left(\sum_{i=1}^{m+1} \delta_{i} \xi_{i}, \beta^{\prime}, 0\right)(\beta+\theta)=\left(\sum_{i=1}^{m+1} \delta_{i} \xi_{i} \beta, \beta^{\prime} \beta, 0\right)$

$\left(\eta, \beta^{\prime}, \theta\right)(\beta+\theta)=\beta^{\prime} \beta+\theta$.

(v) $(\eta, \beta, 0)(\eta+\theta)=0$

$$
\begin{aligned}
&\left(\sum_{i=1}^{m+1} \eta_{i j(i)}, \beta, \theta\right)\left(\sum_{i=1}^{m+1} \delta_{i} \xi_{i}+\theta\right)=\left(\sum_{i=1}^{m+1} \delta_{i} \xi_{j(i)+1}, 0, \theta\right) . \\
& \text { (vi) }\left(\sum_{i=1}^{m+1} \delta_{i} \xi_{i}, \beta^{\prime}, 0\right)(\eta+\beta)=\left(\sum_{i=1}^{m+1} \delta_{i} \xi_{i} \beta, \beta^{\prime} \beta, 0\right) \\
&\left(\sum_{i=1}^{m+1} \eta_{i(j) i}, \beta^{\prime}, \theta\right)\left(\sum_{i=1}^{m+1} \delta_{i} \xi_{i}+\beta\right)=\left(\sum_{i=1}^{m+1} \delta_{i} \xi_{j(i)+1}+\beta^{\prime} \beta\right) .
\end{aligned}
$$




$$
\text { (vii) } \begin{aligned}
& \left(\sum_{i=1}^{m+1} \delta_{i} \xi_{i}, \beta^{\prime}, 0\right)(\eta+\beta+\theta)=\left(\sum_{i=1}^{m+1} \delta_{i} \xi_{i} \beta, \beta^{\prime} \beta, 0\right) \\
& \left(\sum_{i=1}^{m+1} \eta_{i j(i)}, \beta^{\prime}, \theta\right)\left(\sum_{i=1}^{m+1} \delta_{i} \xi_{i}, \beta, \theta\right)=\left(\sum_{i=1}^{m+1} \delta_{i} \xi_{j(i)+1}, \beta^{\prime} \beta, \theta\right) .
\end{aligned}
$$

Proof. Just apply Theorem 2.4.

To simplify the classification, we look first at a case which does not resemble those described above.

THEOREM 6.3. All subgroups of $\theta R$ are left $R$-subgroups of $R$.

Proof. Obvious from Lemma 6.2(i).

Note that such a subgroup consists of elements from the set

$$
\left\{0, \theta, \sum_{i=1}^{m+1} \delta_{i} \xi_{i}, \sum_{i=1}^{m+1} \delta_{i} \xi_{i}+\theta\right\}
$$

where $\delta_{i} \xi_{i}=\delta_{j} \xi_{j}$ for all $i, j, 1 \leqslant i, j \leqslant m+1$. Then we could use (iii) and (v) of Lemma 6.2, if we wished.

Because of Theorem 6.3, we define three subgroups associated with each left $R$ subgroup $K$ of $R$, which we call $K_{1}, K_{2}, K_{3}$.

$$
K_{1}=H K, \quad K_{2}=(G-H)\left(K \cap \delta_{1} M_{0}(H)\right), \quad K_{3}=G(K \cap \theta R) .
$$

The fact that $K_{1}, K_{2}$ and $K_{3}$ are subgroups is easy to check. Note that $K_{1}$ and $K_{2}$ are subgroups of $H$, while $K_{3}$ is a subgroup of $G$.

LEMma 6.4. Let $K$ be a left $R$-subgroup of $R$.Then $K_{2}$ is a normal subgroup of $K_{3}$, and in particular $K_{2} \subseteq K_{3}$.

ProOf. From Lemma 6.2(iii), it is obvious that if $\delta_{i} \xi \in K$ then $\delta_{j} \xi \in K$ also for any $j, \quad 1 \leqslant j \leqslant m+1, \quad \xi \in M_{0}(H)$. Hence $K_{2}=(G-H)\left(K \cap \delta_{i} M_{0}(H)\right)$ for any $i$, $1 \leqslant i \leqslant m+1$. Now let $h \in K_{2}, g \in K_{3}$. We first show that $h \in K_{3}$. We know that there exists $\xi \in M_{0}(H)$ such that $g_{m+1} \delta_{1} \xi=h$. By above $\delta_{i} \xi \in \mathrm{K}$. So $\sum_{i=1}^{m+1} \delta_{i} \xi \in K \cap \theta R$ and thus $h \in K_{3}$. So, in the notation of Theorem 5.3, $\theta_{g} \in K$. Then $-\theta_{g}+\delta_{1} \xi+\theta_{g}$ maps $g_{m+1}$ to $-g+h+g$, and $g_{m+i}$ to 0 for $i>1$. Thus $-g+h+g \in K_{2}$. This suffices to prove the result.

We call a triple $\left(K_{1}, K_{2}, K_{3}\right)$ of subgroups of $G$ admissible if $K_{1}$ and $K_{2}$ are contained in $H$ and $K_{2}$ is a normal subgroup of $K_{3}$. Lemma 6.4 shows that every left $R$-subgroup gives rise to an admissible triple. We now establish the converse : every admissible triple arises from a left $R$-subgroup and the correspondence is one-toone. Given an admissible triple $\left(K_{1}, K_{2} . K_{3}\right)$, let $K \subseteq R$ be defined by

$$
K=\left\{\beta+\eta+\theta_{x} ; H \beta \subseteq K_{1}, G \eta \subseteq K_{2}, \theta_{x}, x \in K_{3}\right\}
$$


Lemma 6.5. $K$ as defined in (6.2) is a left $R$-subgroup of $R$.

Proof. Let $\beta_{1}+\eta_{1}+\theta_{x}$ and $\beta_{2}+\eta_{2}+\theta_{y}$ lie in $K$. Since $K_{1}$ is a subgroup, it follows that $H\left(\beta_{1}-\beta_{2}\right) \subseteq K_{1}$. Also

$$
\beta_{1}+\eta_{1}+\theta_{x}-\left(\beta_{2}+\eta_{2}+\theta_{y}\right)=\beta_{1}-\beta_{2}+\left(\eta_{1}+\theta_{x}\right)-\left(\eta_{2}+\theta_{y}\right) .
$$

We now consider the second part of this expression. So

$$
\begin{aligned}
\eta_{1}+\theta_{x}-\left(\eta_{2}+\theta_{y}\right) & =\eta_{1}+\theta_{x}-\theta_{y}-\eta_{2}+\theta_{y}-\theta_{x}+\theta_{x}-\theta_{y}, \\
& =\eta_{1}+\theta_{x-y}-\eta_{2}-\theta_{x-y}+\theta_{x-y} \text { as } \theta_{x}-\theta_{y}=\theta_{x-y} .
\end{aligned}
$$

Put $x-y=z$. Then

$$
\eta_{1}+\theta_{x}-\left(\eta_{2}+\theta_{y}\right)=\eta_{1}+\theta_{z}-\eta_{2}-\theta_{z}+\theta_{z}
$$

As $K_{3}$ is a subgroup $z \in K_{3}$. Also $g\left(\eta_{1}+\theta_{z}-\eta_{2}-\theta_{z}\right)=g \eta_{1}+z-g \eta_{2}-z$ if $g \in G-H$ and is in $K_{2}$ as $K_{2}$ is a normal subgroup of $K_{3}$. If $g \in H, g\left(\eta_{1}+\theta_{z}-\eta_{2}-\theta_{z}\right)=0$. So $G\left(\eta_{1}+\theta_{z}-\eta_{2}-\theta_{3}\right) \subseteq K_{2}$. This finishes the proof that $K$ is a subgroup. Let $\gamma \in R$. Then $H \gamma \subseteq H$ gives us $H \gamma \beta \subseteq H \beta \subseteq K_{1}, G \gamma \eta \subseteq G \eta \subseteq K_{2}$. Also $\gamma \theta_{x}=0$ or $\theta_{x}$, since $\theta_{x} \in \theta R$ and $\gamma \theta=0$ or $\theta$. This shows that $R K \subseteq K$. Hence the result is proved.

We now come to the final step.

THEOREM 6.6. There is a one-to-one correspondence between admissible triples and left $R$-subgroups of $R$.

ProOf. Let $T$ be the set of admissible triples, $L$ the set of left $R$-subgroups of $R$. We define $\psi: T \rightarrow L$ given by (6.2) and $\varphi: L \rightarrow T$ given by (6.1). It is obvious that $\psi \varphi$ is the identity map on $T$. We will now show that $\varphi \psi$ is the identity map on $L$. So let $K \in L, K \varphi=\left(K_{1}, K_{2}, K_{3}\right), K \varphi \psi=K^{\prime}$.

Since $\left(0, \beta^{\prime}, 0\right)(\eta, \beta, \alpha)=\left(0, \beta^{\prime} \beta, 0\right)$ by Theorem 2.4 , it follows that

$$
K=\left(K \cap M_{0}(H)\right)+\left(K \cap\left(N+Z_{2}\right)\right),
$$

and similarly for $K^{\prime}$. By Theorem 6.1, it follows that

$$
K \cap M_{0}(H)=K^{\prime} \cap M_{0}(H) .
$$

Alternatively the same method that we will apply now can be used to show that $K \cap M_{0}(H)=K^{\prime} \cap M_{0}(H)$. Let $\eta \in K, \eta=\sum_{i=1}^{m+1} \delta_{i} \xi_{i}$. If $g_{1} \xi_{i}=g_{1} \xi_{j}$ for all $i, j, 1 \leqslant i$, $j \leqslant m+1$, then $\eta \in K \cap \theta R$. From the definitions it is immediate that $K^{\prime} \cap \theta R=K \cap \theta R$. So consider $\eta \in K-\theta R$. Then $\eta=\sum_{i=1}^{m+1} \delta_{i} \xi_{i}$ and for some $k \neq j$ we have $g_{1} \xi_{k} \neq g_{1} \xi_{j}$. Let $g_{1} \xi_{k}=h_{k}, g_{1} \xi_{j}=h_{j}$. Then from Lemma 6.2(iii) it follows that $\theta_{x} \in K$ for $x=h_{k}, x=h_{j}$. Then $y-\theta_{x}=\sum_{i=1}^{m+1} \delta_{i}\left(\xi_{i}-\xi_{k}\right)$. Again applying Lemma 6.2(iii) for a suitable choice of $j(i)$, namely $j(l)=k-1$ for $1 \leqslant l \leqslant m, j(0)=j-1$, we 
obtain that $\delta_{1}\left(\xi_{j}-\xi_{k}\right) \in K$. Hence $h_{j}-h_{k} \in K_{2}$, and $h_{j} \in K_{3}$. Similarly we can show that $\delta_{1}\left(\xi_{l}-\xi_{k}\right) \in K$ and thus that $G \delta_{1}\left(\xi_{l}-\xi_{k}\right) \in K_{2}$ for all $l, 1 \leqslant l \leqslant m+1$. Thus $\eta=$ $\sum_{i=1}^{m+1} \delta_{i}\left(\xi_{i}-\xi_{k}\right)+\theta_{x} \in K^{\prime}$, since $G \sum_{i=1}^{m+1} \delta_{i}\left(\xi_{i}-\xi_{k}\right) \subseteq K_{2}$. Thus $K \subseteq K^{\prime}$. But if $G \eta \in K_{2}$, and $\eta=\sum_{i=1}^{m+1} \delta_{i} \xi_{i}$, then $g_{m+i} \delta_{i} \xi_{i} \in K_{2}$. So we can find $\delta_{1} \xi_{i}^{\prime} \in K$ such that $g_{m+1} \delta_{1} \xi_{i}^{\prime}=g_{m+i} \delta_{i} \xi_{i}$ and then $\delta_{i} \xi_{i}=\delta_{i} \xi_{i}^{\prime}$. By the remarks in the proof of Lemma 6.4 , or by Lemma 6.2(iii),

$$
\sum_{i=1}^{m+1} \delta_{i} \xi_{i}^{\prime}=\sum_{i=1}^{m+1} \delta_{i} \xi_{i} \in K .
$$

Hence $\eta \in K$. This, together with the facts proved above, that $K \cap M_{0}(H)=K^{\prime} \cap M_{0}(H)$ and $K \cap \theta R=K^{\prime} \cap \theta R$, show that $K^{\prime} \subseteq K$. Thus $K=K^{\prime}$. This finishes the proof.

COROLlaRY 6.7. If we define $T$ as a lattice in the natural way, the correspondence given in Theorem 6.6 is a iattice isomorphism between $T$ and $L$.

PROOF. This is obvious from the definitions.

THEOREM 6.8. The only two-sided $R$-subgroups of $R$ are the following:

$$
\{0\}, \quad N, \quad N+Z_{2}, \quad M_{0}(H), \quad M_{0}(H)+N, \quad\left\{0_{x} ; x \in K\right\}, \quad R
$$

where $K$ is a subgroup of $G$.

This follows easily from the above analysis. They correspond respectively to the triples :

$$
(0,0,0), \quad(0, H, H), \quad(0, H, G), \quad(H, 0,0), \quad(H, H, H), \quad(0.0, K), \quad(H, H, G) .
$$

\section{References}

G. Betsch (1973), 'Some structure theorems on 2-primitive near-rings', in Rings, modules and radicals, Colloquia Mathematica Societatis János Bolyai 6 edited by A. Kertész. pp. 73-102 (North Holland).

Y. Fong (1979), The endomorphism near-rings of the symmetric groups (Ph.D. Thesis, University of Edinburgh).

H. E. Heatherly (1972), 'One-sided ideals in near-rings of transformations', J. Austral. Math. Soc. 13 , $171-179$.

R. R. Laxton (1963), 'Primitive distributively generated near-rings', J. London Math. Soc. 38, $40-49$.

C. G. Lyons (1973), 'On decompositions of E(G)', Rocky Mountain J. Math. 3, 575-582.

C. G. Lyons and J. D. P. Meldrum (1980a), 'Reduction theorems for endomorphism near-rings' Monatshefte für Mathematik (to appear).

C. G. Lyons and J. D. P. Meldrum (1980b), 'N-series and tame near-rings'. Proc. Roy. Soc. Edinhurah Sect. $A$ (to appear). 
J. J. Malone (1973), 'Generalized quaternion groups and distributively generated near-rings', Proc. Edinburgh Math. Soc. 18, 235-238.

J. J. Malone (1977), 'More on groups in which each element commutes with its endomorphic image', Proc. Amer. Math. Soc. 65, 209-214.

J. J. Malone (1980), 'A non-abelian 2-group whose endomorphisms generate a ring, and other examples of E-groups', Proc. Edinburgh Math. Soc. (to appear).

J. J. Malone and C. G. Lyons (1970), 'Endomorphism near-rings', Proc. Edinburgh Math. Soc. 17, 71-78.

J. J. Malone and C. G. Lyons (1972), 'Finite dihedral groups and d.g. near-rings I', Compositio Math. 24, 305-312.

J. J. Malone and C. G. Lyons (1973), 'Finite dihedral groups and d.g. near-rings II', Compositio Math. 26, 249-259.

J. J. Malone and B. McQuarrie (1970), 'Endomorphism rings of non-abelian groups', Bull. Austral. Math. Soc. 3, 349--352.

J. D. P. Meldrum (1978), 'On the structure of morphism near-rings', Proc. Roy. Soc. Edinburgh Sect A 81 , 287-298.

J. D. P. Meldrum (1979), 'The endomorphism near-rings of finite general linear groups', Proc. Roy. Irish Acad. Sect. A 79, 87-96.

J. D. P. Meldrum and C. G. Lyons (1978), 'Characterizing series for faithful d.g. near-rings', Proc. Amer. Math. Soc. 72, 221-227.

G. Pilz (1977), Near-rings (North-Holland, Amsterdam; American Elsevier, New York).

W. R. Scott (1964), Group theory (Prentice-Hall, Englewood Cliffs, N.J.).

\section{Department of Mathematics}

King's Buildings

Mayfield Road

Edinburgh EH9 3JZ

U.K. 